\title{
Die chinesische Presse der Jahrhundertwende im internationalen Kontext
}

\author{
NATASCHA GENTZ
}

Der vorliegende Beitrag befaßt sich mit der Internationalisierung von Presse und Medien um die Jahrhundertwende aus einer globalen Perspektive, nämlich den Einflüssen, Auseinandersetzungen und Hintergründen der Entstehung einer nationalen chinesischen Presse im transnationalen Kontext. Zweifellos war um die Jahrhundertwende China aufgrund verschiedenster kultureller, diplomatischer und politischer Begegnungen über die letzten Jahrzehnte ein beliebtes Thema in europäischen Kulturzeitschriften geworden. Wenig allerdings ist darüber bekannt, wie chinesische Journalisten in Zusammenarbeit mit ausländischen Journalisten an der Konstruktion und Verbreitung von ChinaBildern in Europa beteiligt gewesen sein mochten.

Das liegt zum einen daran, daß die Frühphase des modernen chinesischen Journalismus im ausgehenden 19. Jahrhundert über lange Zeit von der Forschung vernachlässigt worden ist, da die ersten Publikationen und Tageszeitungen als triviale und unbedeutende Unterhaltungsblätter eingeschätzt wurden, denen keine soziale und politische Bedeutung zukamen. Es herrschten gegenüber der Entstehung und Verbreitung moderner chinesischer Medien in der Mediengeschichtsforschung auch weitere Vorurteile, die es auszuräumen gilt, wenn man die tatsächlichen Verflechtungen von Interaktionen und Wirkungsfeldern der neuen Journalisten zu verstehen sucht. Dazu gehört die Auffassung, daß die Entstehung einer modernen chinesischen Presse allein im Import westlicher, d. h. zunächst europäischer Medientechnologien gründete; daß nur die Extraterritorialität der Konzessionsgebiete in den Metropolen eine liberale britische Pressefreiheit garantierte, während die indigene imperiale chinesische Regierungsmacht jede Entfaltung solcher öffentlicher Kommunikation zu verhindern suchte; daß eine chinesischsprachige Presse wesensgemäß eine pro-chinesische, patriotische Haltung einnehmen würde, während die ausländische Presse in China die Interessen der Ausländer vertrete.

Diese vornehmlich politischen und ideologischen Interpretationen der Inhalte der Zeitungen basieren auf herkömmlichen kolonialgeschichtlichen Annahmen über die Begegnungen zwischen China und dem Westen, die unter den Stichwörtern von ,Imperialismus' und ,Kanonenbootpolitik' zusammengefaßt werden können. Demnach haben westliche, zunächst vornehmlich europäische, Mächte die Akzeptanz der westlichen zivilisatorischen Überlegenheit 
durch militärische Gewalt auferzwungen, während China aufgrund innerer Schwäche und politischer Krise nicht imstande war, dem Angriff der imperialistischen Mächte zu widerstehen. Daß sich diese Vereinfachungen in den tatsächlichen komplexen politischen, kulturellen und sozialen Auseinandersetzungen nicht aufrecht erhalten lassen, läßt sich gerade im Bereich des neuen Journalismus besonders verdeutlichen.

Wie in Europa, erlebten Formen von öffentlicher Artikulation, Kommunikation und Information auch in China besonders im 19. Jahrhundert eine enorme Veränderung, bedingt durch technische, soziale und politische Entwicklungen und das Entstehen eines neuen Informationsbedarfs über die Vorgänge in der gesamten Welt. Die periodische Publikation bzw. die Tageszeitung waren in China wie in Europa innerhalb kurzer Zeit das dominante Medium neuer gesellschaftlicher Diskurse und eines neuen internationalen Nachrichtenhandels geworden.

Die Zeitung in China konnte eine solche Durchsetzungskraft entfalten, da die chinesischen Zeitungsmacher sich sowohl an modernen Zeitungsmodellen orientierten wie sie sich gleichzeitig indigene Kommunikationsstrukturen zunutze machten. Als transnationales Medium, das sie von Anfang an war, stand die chinesische Presse im Spannungsfeld der Auseinandersetzung mit einer neu wahrgenommenen Welt, mit einer auferzwungenen ausländischen Präsenz in China und kritischen Reflexionen über das eigene kulturelle Erbe.

Durch diesen hybriden Charakter wurde die neue Presse somit zum Verhandlungsort der zentralen kulturellen, politischen, wirtschaftlichen und gesellschaftlichen Fragen der späten Qing-Zeit, was ebenfalls die relativ schnelle Akzeptanz des neuen Mediums in China erklärt.

Die ersten chinesischen Zeitungen und Zeitschriften entstanden in den rasch wachsenden Metropolen Hongkong und Shanghai seit Mitte des 19. Jahrhunderts, und somit in Hafenstädten, die durch die Opiumverträge seit den 1840er Jahren dem internationalen Handel geöffnet wurden. Als internationale Handelszentren und als Auffangbecken für Zuwanderer unterschiedlichster sozialer Schichten aus dem Hinterland lassen sich diese Orte als kulturelle Experimentierfelder beschreiben: Hier entstanden neue Institutionen zur Organisation der neuen Gemeinschaft, - Assoziationen, Museen, Bibliotheken und Bildungsvereine - sowie neue Medien zur Vermittlung neuen Wissens, neuer Werte und der neuen Ordnung. Neue Arbeitsmärkte erforderten neue Qualifikationen, die sich stark von den traditionellen Bildungswegen unterschieden.

Der folgende Beitrag sucht anhand einiger Beispiele einen Einblick in diese dramatischen Transformationen zu vermitteln. Zunächst ist zu fragen, wie eine moderne Presse, die zweifellos aus dem Westen nach China gelangt war, auf chinesischem Boden Fuß fassen konnte und mit welchen Strategien sich die neuen Journalisten auf dem Pressemarkt zu etablieren suchten. Im weiteren wird sich der Beitrag mit den spezifischen Produktionsbedingungen beschäfti- 
gen, sowie mit den Inhalten und der Akzeptanz des frühen chinesischen Journalismus.

Als Anfang einer modernen chinesischen Presse wird häufig die Gründung des Chinese Monthly Magazine (Chashisu meiyue tongjizhuan) genannt, das 1815 von den britischen Missionaren der presbyterianischen London Missionary Society (LMS) und eigentlichen Gründern der protestantischen Mission in China, Robert Morrison (1782-1834) und William Milne (1785-1822), in Malacca ins Leben gerufen wurde. ${ }^{1}$ Das protestantische Projekt, Mission eher über das gedruckte Wort denn über Ritual und Performanz auszuüben, hatte nicht nur in der Reformationszeit die Enwicklung des Buchmarktes wesentlich vorangetrieben, sondern auch in China den technischen Fortschritt des Pressewesens angestoßen, z. B die Entwicklung technischer Verfahren zur Herstellung von beweglichen Lettern, den Import westlicher Druckmaschinen und später die Einfuihrung der Lithographietechnik. Die ersten Meilensteine der technischen Entwicklung wurden hierbei sämtlich von britischen und amerikanischen Presbyterianern gesetzt - angefangen mit dem Druck eines ersten chinesischenglischen Lexikons von Robert Morrison 1815 bzw. dessen erster Bibelübersetzung.

Die hierfür hergestellten Letternsätze wurden weiter zum Druck des oben genannten ersten missionarischen Magazins Chinese Monthly Magazine benutzt, und diese Verlagspresse wurde wiederum Vorbild für ebensolche Einrichtungen in Batavia und Macao. Und nicht nur die erste chinesische Tageszeitung Xunhuan Ribao (The Universal Circulating Herald) wurde mit Hilfe der Druckmaschinen und Einrichtungen der LMS hergestellt, sondern auch viele der chinesischen Ausgaben der ersten britischen Zeitungen wurden dann ins Leben gerufen, wenn ein Missionsverlag einen Satz von chinesischen Lettern zum Verkauf bereitgestellt hatte. So hatten Missionare schon in den 60er Jahren des 19. Jahrhunderts eine Monopolstellung im modernen Druck chinesischer Bücher erlangt.

In Hongkong operierten zunächst zwei Missionarspressen, von denen die bekanntere, die Druckerei des Englisch-Chinesischen Kollegs (Yinghua shuүuan), erstmals chinesische Mitarbeiter einstellte. ${ }^{2} \mathrm{Zu}$ den ersten Journalisten in China zählten somit berühmte Missionare wie James Legge (chines. Li Yage 1814-1897) oder Ernst Eitel (chines. Ai De, 1838-1908). James Legge gilt auch als Begründer der Sinologie in Großbritannien, da er mit dem Gelehrten Wang Tao (1828-1897) die chinesischen Klassiker ins Englische übersetzte

$1 \mathrm{Zu}$ Kurzbiographien und Listen der Veröffentlichungen der beiden Missionare in China siehe Memorials of Protestant Missionaries to the Chinese, S. 3-24.

2 Fang Hanqi, S. 293 ff. bzw. Guo Weidong, S. 199. Auch französische Missionare gründeten Druckereien, z. B. die Imprimerie de Nazareth (Nazale yinshuguan), die 1884 von französischen Katholiken gegründet wurde. Ebd., S. 234. 
und damit eine Ausgabe schuf, die heute noch oftmals zitiert wird. ${ }^{3}$ Die erste Missionarspresse im Inland Chinas wird 1830 in Kanton von dem professionellen Drucker Wells Williams ins Leben gerufen, gefolgt von der Gründung der American Presbyterian Mission durch Walter M. Lowrie 1844 in Ningbo; nach seinem Umzug nach Shanghai avancierte dieser Amerikanisch-Chinesische Verlag (Meihua Shuguan) in den 60er Jahren zum erfolgreichsten Großunternehmen im Druckbereich, das über 100 Arbeiter angestellt haben soll. ${ }^{4}$

Missionare wirkten als Kulturvermittler, und so wurde das europäische Chinabild im 19. Jahrhundert wesentlich von den zahlreichen Berichten dieser Missionare geformt und weitergegeben. Umgekehrt enthielten viele der ersten missionarischen Publikationen einen erheblichen Anteil an Darstellungen über den Westen und Einführungen in die z. T. ebenso in Europa brandneuen westlichen Naturwissenschaften. So behandelte das 1868 von Young J. Allen in Shanghai gegründete Magazin Church News (Jiaohui Xinbao) zwar dem Titel entsprechend zu etwa $30 \%$ religiöse Themen, der überwiegende Teil von 60 $\%$ war jedoch säkularen Nachrichten aus aller Welt und naturwissenschaftlichem und technischem Material gewidmet. Diese Missionarszeitungen stellten somit die ersten Kulturzeitschriften und Wissenschaftsmagazine in China. ${ }^{5}$

Als Wang Tao 1874 die Xunhuan Ribao ins Leben rief, hatte sich das ausländische Publikationswesen in Hongkong mit portugiesischen Druckereien, britischen Verlagshäusern und missionarischen Druckunternehmen schon über Jahrzehnte etabliert. In den 1870er Jahren verlagerte sich das Zentrum des nationalen Buchmarktes schließlich völlig nach Shanghai, wofür vor allem die Einführung der lithographischen Drucktechnik verantwortlich gemacht wird. Lithographien wurden seit ihrer Erfindung im späten 18. Jahrhundert schnell zu einer verbreiteten Reproduktionstechnik in Europa, die in berühmten Illustrierten, wie Harper's The Graphic oder Le Monde Illustré Anwendung fand. ${ }^{6}$ Die neue Technologie wurde auch von Missionaren genutzt, die sie überhaupt erstmals in Shanghai in der jesuitischen Missionsstation in Xujiahui einführten, und von John Fryer (1839-1928) in der ersten chinesischen Tageszeitung Shanghais, der Shanghai Xinbao (1862-1872), sehr erfolgreich eingesetzt. Den Durchbruch mit dieser Technologie in Shanghai schaffte aber das ShenbaoHaus.

$3 \mathrm{Zu}$ Wang Tao siehe Cohen.

4 Barnett; Ji Shaofu, S. 264-267.

5 Es handelt sich hier um den ebenfalls sehr erfolgreichen und weit rezipierten Vorläufer der oben erwähnten Globe Magazine. Siehe die inhaltliche Analyse der Zeitschrift in Kapitel 4 in Bennett.

6 Lithographien ermöglichten erstmals eine möglichst genaue Reproduktion von Bildern und Handschriften und boten eine billige Alternative zu den zeit- und kostenaufwendigen Herstellungen der Druckstöcke. 
Die Shenbao (1872-1949) ${ }^{7}$ wurde 1872 zu einem Zeitpunkt ins Leben gerufen, da sich schon einige ausländische Zeitungen fest etabliert hatten, allen voran die britische Wochenzeitung North China Herald (Beihua jiebao 18501951), 1850 von Henry Shearman gegründet, mit der 1864 folgenden Tagesausgabe der North China Daily News (Zilin xibao, 1864-1951). Daneben existierten einige kleinere und sehr kurzlebige Zeitungen wie die Shanghai Daily Times, die Shanghai Recorder oder Shanghai Evening Express. Nur die Celestial Empire (zusammen mit dem Shanghai Mercury) konnte an die Bedeutung des North China Herald heranreichen. ${ }^{8}$ Wie R. G. Wagner zeigte, trat Ernest Major (Meicha, 1841-1908) sowohl mit seiner Zeitung als auch dem parallel laufenden Buchverlag genau in die Lücke, welche die Verwüstungen der Taiping Rebellion hinterlassen hatten, indem er populäre und verlorengegangene chinesische Bücher suchen ließ und nach den alten Vorlagen mit der neuen Drucktechnik neu publizierte und damit äußerst erfolgreich war. ${ }^{9}$

Die chinesischen Ausgaben der Zeitungen wurden meist in Kooperation mit chinesischen Konvertiten erstellt. Wie verschiedene Überlieferungen berichten, wurde den chinesischen Literaten dabei der Inhalt des Textes von den Missionaren auf Chinesisch erklärt und von jenen dann in flüssigem schriftlichem Chinesisch niedergelegt. ${ }^{10}$ Einige dieser Mitarbeiter avancierten später zu eigenständigen Journalisten von großen Tageszeitungen, wie z. B der oben erwähnte Wang Tao.

Die missionarischen Verlagshäuser wurden durch ihre Publikationen und ihre Funktion als internationale Treffpunkte zu Zentren der Vermittlung zwischen Ost und West: situiert in den großen Hafenstädten wie Shanghai oder Hongkong, waren sie Informations- und Anlaufstelle für Durchreisende und Ausgangspunkt für erste Überseereisende geworden. Wie aus zeitgenössichen Tagebüchern oder Reiseaufzeichnungen von z. B. Guo Songtao (1818-1891), Li Gui (1842-1903) oder Ma Jianzhong (1845-1899) ersichtlich, wurde auch das Verlagshaus der Xunhuan Ribao ein wichtiges Zentrum, welches man auf-

7 Die Shenbao war die größte und langlebigste nationale Tageszeitung in China. Nach Gründung der Volksrepublik wurde sie aufgelöst und von da an in ihren Verlagshäusern in der Hankoulu in Shanghai die „Befreiungszeitung“ (Jiefang Ribao) herausgegeben.

8. Für einen Überblick über die britischen, aber auch französischen, deutschen, portugiesischen et. al. Zeitungen siehe King / Clarke, S. 76-97. Die später oft zitierte Tageszeitung The Celestial Empire wurde 1874 von dem Portugiesen Pedro Loureiro gegründet und war Teil der Shanghai Mercury Gruppe. Die Gruppe hatte ein weites Korrespondentennetz und rühmte sich, besonders unabhängig zu sein. Ebd., S. 87-88.

9 Ausführlich zu Majors lithographischen Produkten, Verlagsaktivitäten und Marktstrategien siehe Wagner.

10 Xiong Yuezhi, S. 475-480. Die Bibelübersetzung Wang Tao's, die auf diese Weise in Kooperation mit Henry Medhurst entstanden ist, wird aufgrund ihres feinen literarischen Stils die populärste und einflußreichste Bibel des 19. Jahrhunderts genannt. Vgl. Lo Hsiang-lin, S. 43 und 45. 
suchte, um sich vor internationalen Reisen zu informieren. In diesen Institutionen hielten sich ja nicht nur zum Teil selbst Auslands- und reiseerfahrene Journalisten auf - die zahlreichen Buchpublikationen über die Verhältnisse im Ausland wurden auch als nützliche Reiselektüre an diesen Orten erworben. (Zhou Jiarong, S. 1-9)

Auch viele der fortschrittlichsten chinesischen Beamten hielten schon früh Kontakt zu Missionaren und Journalisten. Der bekannte Reformer Feng Guifen (1809-1874) traf sich zum Beispiel schon in den 1860er Jahren einmal wöchentlich mit dem amerikanischen Missionar und Journalisten Young Allen (chines. Lin Lezhi, 1836-1907) und war wiederum enger Freund der Journalisten der Shenbao und Wang Taos. Der britische Missionar und Mitherausgeber der einflußreichsten Missionars-Zeitschrift Globe Magazine (Wanguo gongbao), Timothy Richard (1845-1919), oder der amerikanische Gilbert Reid (1857-1927) waren zum Ende des 19. Jahrhunderts soweit in die Nähe des politischen Zentrums gerückt, daß sie 1898 eine einflußreiche Rolle in der berühmten und kurzen Reformbewegung des Guangxu-Kaisers spielen konnten. ${ }^{11}$ Missionarische Mitarbeiter an Regierungsprojekten, wie Young Allen am Shanghaier Übersetzerbüro der Regierung (Guangfang yanguan) oder John Fryer (chines. Fu Lanya, 1839-1928) am Shanghai Arsenal, wurden zudem von der chinesischen Regierung mit Titeln und Symbolen der chinesischen Beamtenhierarchie ausgezeichnet. ${ }^{12}$

Diese neuen chinesischen Journalisten, die sich mit ihrer westlichen Schuloder Berufsausbildung in den Missionarsschulen den traditionellen Karrierewegen über die Staatsexamina entzogen hatten, waren somit in Hongkong und Shanghai schnell zum Bestandteil einer neuen sozialen Elite aufgestiegen. (Smith, S. 29-56) In seinen Erinnerungen an die späteren Jahrzehnte in Hongkong ist Wang Tao voll des Lobes über die westlichen Errungenschaften und Einrichtungen der Kolonie und seinen freundschaftlichen Umgang mit James Legge. ${ }^{13}$ Im Jahre 1872 kehrt er von einer Europareise mit jenem heute bekanntesten Sinologen nach Hongkong zurück und wird in den Hongkonger Zeitungen aufgrund seiner Expertise in westlichem Wissen respektvoll mit ,Dr. Wong' tituliert (Sinn, S. 10 f.).

Eine prosopographische Studie von etwa 150 Journalisten aus dem Zeitraum von 1860 bis 1911 zeigt, daß wir es insgesamt mit einer relativ homogenen Gruppe zu tun haben, die sehr ähnliche biographische Elemente mit Wang Tao teilen: Meist hatten die Journalisten den ersten Prüfungsrang erworben, sich dann aber von diesem Bildungsweg abgewandt, sie hielten engen

11 Z. B. Soothill; Tsou Mingteh, S. 73-90.

12 Bennett 1983 sowie 1967. Fryer wurde zwar trotz eigener Bemühungen nie in eine Missionsgesellschaft aufgenommen, stand aber in sehr engem Kontakt zu den Missionaren in Shanghai und nahm an deren Konferenzen teil.

$13 \mathrm{Zu}$ zwiespältigen Ansichten zu Hongkong von Wang Tao et.al. siehe Vittinghoff, S. 23-24. 
Kontakt zu Missionaren oder deren Bildungseinrichtungen, waren zum großen Teil einer Fremdsprache mächtig oder sogar schon ins Ausland gereist. Der Großteil von ihnen waren Neuankömmlinge in den Metropolen, was eine schnelle Netzwerkbildung untereinander beförderte. Die enge Kooperation zwischen ausländischen und chinesischen Journalisten über den gesamten Zeitraum von 1870 bis in das frühe erste Jahrzehnt des 20. Jahrhunderts unterstreicht den transnationalen Charakter dieser publizistischen Unternehmungen und bedeutete auch eine konstante Aushandlung westlicher und chinesischer Werte.

Gleichzeitig sahen sich die ersten Journalisten oftmals mit Vorwürfen des konfuzianischen Establishments konfrontiert, die eigenen kulturellen Wurzeln $\mathrm{zu}$ verraten und den Ausverkauf der literarischen Tradition zu betreiben. Im kaiserlichen China oblag die Autorität und das Recht öffentlich zu sprechen allein einer kleinen Elite, die die Stadien des bekanntlich enorm zeitaufwendigen und schwierigen Prüfungssystems gemeistert hatten. Im 19. Jahrhundert waren durch verschiedene Krisen die normativen Grundlagen dieser Literatenklasse wesentlich erschüttert worden. Die Fremdherrschaft der Mandschus verweigerte ihnen oftmals die traditionelle Rolle als politische und moralische Ratgeber der Regierung; die Zahl der vergebenen Prüfungsränge steigerte sich explosionshaft, so daß damit nicht selbstverständlich ein öffentliches Amt verbunden war; gleichzeitig stieg der inoffizielle Verkauf von Prüfungsrängen so inflationär an, daß das Ansehen der Rangträger als Wächter und Bewahrer des kulturellen Erbes unterminiert wurde. Die neue öffentliche Stimme der Journalisten, die nun auf eigenen Wegen eine neue Öffentlichkeit anzusprechen suchten, stellte freilich eine weitere Herausforderung für die Position der etablierten Literaten dar. Wenig erstaunlich ist daher die Tatsache, daß Journalisten oftmals verhöhnt und als Anti-Modell präsentiert wurden:

In politischen Kreisen waren Zeitungen weder respektiert, noch wurden sie stark zu Rate gezogen. Wer auch immer Talent hatte, widmete sich den Prüfungen und weigerte sich, den Journalismus zu seinem Beruf zu machen. Nur Bohemiens oder erfolglose Studenten nutzten gelegentlich die Presse, um Ärger und Frustrationen Luft zu machen. (Lei Jin, S. 27a)

Um solche Äußerungen ins rechte Licht zu rücken, sollte man jedoch auch im Auge behalten, daß auch im Europa des 19. Jahrhunderts ähnlich negative Urteile über Journalisten verbreitet waren. Eine grundsätzliche soziale und moralische Inkompetenz schrieb zum Beispiel schon der politische Journalist Ferdinand Lassalle, der Begründer der sozialdemokratischen Bewegung in Deutschland, den Journalisten in seiner entschiedenen Aburteilung des gesamten Berufsstandes von 1863 zu: „Aber wer will unter dieses Heer [der Zeitungsschreiber] gehen, wer, der sich selber achtet, wer, der nur irgendeine Befähigung zu reellen Leistungen auf dem Gebiete der Wissenschaft, des Gedankens oder des bürgerlichen Lebens in sich fühlt?" (Zit. bei OebsgerRoeder, S. 35) 
Leo Wörl, der sich schon frühzeitig mit der Geschichte und Entwicklung des deutschen Pressewesens beschäftigte, stellte 1881 fest: „Die Publizistik wird zu den unehrlichen, Gewerben' gezählt, d.h. zu denen, die sich außerhalb des sozialen Organismus bewegen, wie ehedem Schäfer, Kesselflicker, Zahnärzte, Zigeuner und Schauspieler." (Ebd., S. 33)

Über die 1893 stattfindende Gründung der Xinwenbao (1893-1941) erinnerte sich der Missionar Samuel I. Woodbridge (1856-1926) Jahrzehnte später:

I remember distinctly when the "Sin Wan Pao' was started early in 1893. At that time there was no journalistic background. In most places no one would read a newspaper even if it were presented as a gift. What concern to the man in the street were the affairs of Canton and Peking? A dead dog or a private brawl in his native street was more interesting to the ordinary Chinese than a big revolution in South Africa, America or even the Province of Yünnan. ( S. 31-32)

Potentiell war mit dem Berufsstand des Journalisten jedoch auch eine Publizität verbunden, die als Sprungbrett für eine höhere Karriere dienen konnte. Wie Jacobi ausführt, war der Übertritt von einem Redaktionsbüro in ein Staatsamt in vielen europäischen Ländern und Amerika durchaus keine Seltenheit. ${ }^{14}$ Diese Möglichkeit ist in sehr ironischer und kritischer Weise in Maupassants Roman Bel-Ami in der Figur des George Duroy repräsentiert, der vor allem über günstige Beziehungen zu einflußreichen Ehefrauen aus dem Nichts zum Medienzar avanciert und sogar in die Nähe eines Ministerpostens rückt. ${ }^{15}$ Der schnelle gesellschaftliche und politische Aufstieg und das damit verbundene Potential, Einfluß auf die Politik oder die Massen nehmen zu können, wurde auch als Gefahr wahrgenommen. Die Möglichkeit des gesellschaftlichen Aufstieges von westlichen Journalisten wird als Wunschbild auch von chinesischen Zeitungsmachern immer wieder zitiert:

Ihr [der Zeitung] Nutzen für die Staatsangelegenheiten ist [im Westen] so groß, daß von den gebildeten und moralischen Herren einige gestern noch Redakteure waren und heute an der Regierung sind; andere sind noch am Morgen aus hohem Staatsamt entlassen und treten abends schon in ein Zeitungshaus ein. ${ }^{16}$

In fast wörtlicher Weise beschrieb auch Lei Jin (1871-1941) in seinen Reminiszenzen an die frühe Shenbao seine Einschätzung des westlichen Journalisten:

Während ich diesen Artikel entwerfe, kommt mir unwillkürlich [folgende] Assoziation dazu: Ich habe gehört, daß die Herren in Europa und Amerika den Journalismus heutzutage wirklich für das höchste und vortrefflichste Gewerbe halten.

14 Jacobi, S. 167. Zu den verschiedenen Bildern von Journalisten in Deutschland, Frankreich und England siehe O’Boyle, S. 291-317.

15 Es sei hier nur erwähnt, daß Maupassants Anliegen nicht die Kritik der französischen Presseszene gewesen ist, sondern das Zeitungshaus stellvertretend für irgendein wirtschaftliches Unternehmen des späten 19. Jahrhundert stehen sollte.

16 Liang Qichao, [1896]. „Lun ribao you yi yu guoshi“ (Über den Nutzen von Zeitungen für die Staatsangelegenheiten). 
Deshalb kommt es vor, daß man morgens noch Ministerpräsident oder Staatspräsident ist und sich abends als Redakteur eines Zeitungshauses zurückzieht. Aber es kann ebenso vorkommen, daß man heute noch Herausgeber einer Zeitung und morgen schon als Ministerpräsident oder General tätig ist. Wie angesehen und wichtig muß doch dieser Status sein! (Lei Jin, S. 28b)

Auf der anderen Seite standen den chinesischen Journalisten eine Reihe von Optionen aus der eigenen kulturellen Tradition als Quellen zur Legitimierung ihres neuen Unterfangens zur Verfügung. Eine Zeitung zu etablieren diente, in China wie im Westen, vornehmlich zwei Zielen: nützliches Wissen an eine breite Öffentlichkeit zu verbreiten und Meinungen an und mit einer, imagined community' zu kommunizieren. Beide Ziele waren bereits Teil der vormodernen Strukturen öffentlicher Kommunikation der Qing Regierung, institutionell realisiert in Form des Zensorates, ideologisch integriert im politischen Ideal eines freien Flusses von Kommunikation zwischen Herrscher und Volk durch reine, aufrechte Beamte. ${ }^{17}$ Die neuen Journalisten verfolgten daher die doppelte Strategie, ihre Unternehmungen einerseits in eine vertraute Rhetorik einzubetten und diese politischen Floskeln andererseits mit neuen Bedeutungen zu füllen.

Diese Einbindung der neuen Form der Zeitung in eine lange Tradition politischer Kommunikation, begründet in den konfuzianischen Klassikern, erfolgte durch zahlreiche Zitate, die belegten, daß auch die Zeitungen allein eine „Verbindung von oben und unten“ darstellten. ${ }^{18}$ Den Lesern wurden Präzedenzfälle präsentiert, beginnend mit vorhistorischen heiligen weisen Herrschern Zao und Shun über Zitate aus Werken des 3. und 4. vorchristlichen Jahrhunderts, die allesamt belegen sollten, daß man auf einem gemeinsam akzeptierten kulturellen Boden operierte bzw. hervorhoben, daß Zeitungen nicht allein ein westlicher Import waren. (Xunhuan Ribao, Feb. 6, 1874) Auch wurden direkte Verbindungen zwischen alter, klassischer Vergangenheit und der Gegenwart gezogen, wie im folgenden:

Die Einrichtung einer Zeitung ist vom Namen her etwas Neues, in der Tat ist es jedoch die Fortsetzung einer Tradition. In alten Zeiten schickten Kaiser Wagen aus, um Gerüchte im Volk zu sammeln. Sie erreichteten Tafeln an den Straßen für Beschwerden und Banner zum Lobpreis. (Wie die Zeitungen) hatten sie zum Ziele, sich über die Ansichten im Volke zu informieren. (Ebd.)

Gleichzeitig und im Kontrast zu dieser Selbstdarstellung operierten die Zeitungen als moderne, kommerzielle Unternehmen, die ihren Lesern eine neue Sicht auf die Welt nahebringen und sie mit neusten technischen, politischen, sozialen und kulturellen Phänomenen aus aller Welt vertraut machen wollten.

17 Z. B. Eastman, S. 595-611; Beatrice Bartlett, 1991.

18 Z. B in „Benqu ribao tongqi (Anzeige unsere Nachrichtenhauses)“, Xunhuan Ribao, Feb. 5, 1874, S. 3. „Lun xinwenzhi zhi yi (Über den Vorteil von Zeitungen)“ (Shenbao, Aug. 11, 1886, S.1.). 
Und dabei orientierten sich die Zeitungen wie ihre Journalisten direkt an westlichen Vorbildern, wenn sie sich in programmatischen Anzeigen zum Ziele setzten, daß ihre Zeitung ,should eventually become in China what the London Times is in England" (China Mail, 2. Feb 1874).

Auch die neuen Institutionen zur Verbreitung westlichen Wissens orientierten sich oftmals direkt an westlichen Vorbildern - so war die Polytechnical Institution auf der Regent's Street in London das Modell des Gezhi shuyuan. Der chinesische Direktor Xu Shou gehörte zu den aktivsten Unterstützern dieser Einrichtung und hatte ambitionierte Pläne: In seiner äußeren Gestaltung sollte eine neu einzurichtende Messehalle dem berühmten Kristallpalast der Londoner Weltausstellung von 1851 gleichkommen, einem großen Komplex aus Glas und Stahl, der durch viele Berichte von chinesischen Diplomaten und Reisenden in Europa bekannt geworden war. ${ }^{19}$ Die akademische Einrichtung war im traditionellen Baustil gehalten. In dieser Institution sollten verschiedene Einrichtungen zur Popularisierung der Wissenschaften vereint werden wie eine Bibliothek, ein naturwissenschaftliches Museum oder öffentliche Vorführungen physikalischer Experimente. Das Institut gab seit 1875 auch ein sehr erfolgreiches wissenschaftliches Magazin, das Wissens-Magazin (Gezhi huibian), heraus, dessen Ziel es war "to make this magazine to China what the SCIENTIFIC AMERICAN is to every country where the English language is known “20. Das Magazin wurde auch durch seine Leserbriefsektion berühmt, in der Fragen zu allen Bereichen des Wissens gestellt und von den Wissenschaftlern beantwortet wurden. ${ }^{21}$

Im modernen Sinne war das Rollenmodell für den Journalisten der Sozialforscher, der Daten und Informationen über die Gesellschaft nach objektiven Kriterien sammelte und verbreitete, und es ist sicherlich kein Zufall, daß dieses Modell zur gleichen Zeit aufkam, als die „Soziologie“ (qunxue) durch Übersetzungen von Yan Fu (1854-1921) und anderen zum viel zitierten Modewort schlechthin avancierte. Gleichzeitig betätigten sich viele Journalisten ebenfalls als Übersetzer und Forscher in jenen neu gegründeten Institutionen, die sich mit westlichem Wissen befaßten. Auch hier kann Wang Tao als Beispiel dienen, der, nachdem er die Redaktion der Xunhuan Ribao verlassen hatte, zum Direktor des oben genannten prominenten Shanghai Polytechnic Institution and Reading Room (Gezhi shuyuan) berufen wurde.

Verläßlich sollten die Quellen sein, und wahrheitsgemäß die Berichterstattung, wie die folgende Jobanzeige demonstriert:

19 Biggerstaff; Lu Yongling / Hayhoe.

20 „The Editor of the Scientific American reviews the Chinese Scientific Magazine and quotes from a letter sent by John Fryer with a sample copy." 29. April 1876. JFA, BL.

21 Die Zeitschrift wurde in über 20 Städten vertrieben - Ende der 1880er Jahre sogar in über 30 und die ersten neun Ausgaben mit einer Auflage von 3000 Exemplaren mußten aufgrund der Nachfrage in einer zweiten Auflage nachgedruckt werden. Li Sanpao, S. 742-44. 
[Der Kandidat] muß über ein breites Wissen und hohe Literarizität verfügen, und vertraut sein mit Gegenwartsthemen. Er muss von hohem moralischen Charakter sein und die Wahrheit in den Tatsachen (shishi qiushi) suchen, um unserem Hause von Nutzen zu sein. (Shenbao, 7. July 1875)

„Die Wahrheit in den Tatsachen suchen“, ist nicht nur ein Zitat, das Deng Xiaoping in den frühen 1980er Jahren zur Grundlage der heutigen chinesischen Reform- und Öffnungspolitik gemacht hat. Es ist auch ein Zitat aus einem der ältesten Geschichtsbücher über die Han Dynastie, dem Hanshu, und wurde seit dem 18. Jahrhundert als Slogan für unparteiliche Gelehrsamkeit angeführt. Als Zusatzqualifikation wurden zudem auch Kenntnisse in Fremdsprachen genannt, denn ein großer Teil der Nachrichten bestand aus Nachdrucken und Übersetzungen aus anderen, oft internationalen Zeitungen. ${ }^{22}$

Vor der Jahrhundertwende zum 20. Jahrhundert hatten alle chinesischen Zeitungen ein ähnliches Layout und eine ähnliche Unterteilung in Kategorien wie Leitartikel, auswärtige Nachrichten, Lokalnachrichten, Übersetzungen von ausländischen Zeitungen, Gedichte und Exzerpte aus anderen (meist Hongkonger) Zeitungen. Die Nachrichten aus Peking wurden in der festgelegten Reihenfolge von kaiserlichen Anordnungen, dann Throneingaben und schließlich Ausschnitten aus den Yamen-Nachrichten aus Jiangsu und Zhejiang gedruckt. Ein großer Teil der Beiträge waren, neben Nachdrucken, Zusendungen von Lesern oder unterhaltsame Geschichten, die auch erfunden und somit am Schreibtisch produziert sein konnten. Der Journalist, der sich auf der Suche nach Nachrichten die Schuhsohlen durchlief, war noch keine häufige Erscheinung unter den Redakteuren. ${ }^{23}$ Gleichwohl war den Artikeln der Shenbao zu entnehmen, daß ein großer Teil der Nachrichten auf der Straße recherchiert wurde bzw. in den Teehäusern der Stadt. Die neuen Teehäuser und Gärten - allen voran der berühmte Zhang Yuan Garten in Shanghai - sind als Räume einer neuen bürgerlichen Öffentlichkeit beschrieben worden, in welchen Möglichkeiten zur Artikulation und zum Austausch von neuen Ideen geboten und genutzt wurden. Auch privat wurden diese Lokalitäten von den Journalisten häufig frequentiert, dort feierten sie Geburtstage oder hielten Ehren-Bankette ab. Sie boten aber auch Raum für Treffen von Assoziationen, Studiengruppen oder politische Agitation. ${ }^{24}$ Zudem besuchten die Redakteure

22 „Yan qing tongren“ (Übersetzer gesucht), Shenbao, 20 Feb 1877. Der hier gesuchte „Übersetzer“ sollte laut Anzeige auch Artikel und Nachrichten verfassen.

23 Die durchgelaufenen Schuhsohlen waren nicht nur in Europa ein symbolisches Bild für den umtriebigen Journalisten, sondern zierten zum Beispiel auch die Titelseiten von chinesischen Journalistenzeitschriften seit den 1930er Jahren. Siehe z. B. das Titelbild ,Jizhe zhe xie (Die Schuhe der Journalisten)“, Baoxue zazhi, vol. 1. no. 10 (Januar 1949).

24 Z. B. Yu Qian über den Yu-Garten oder Jing Cuan über den Zhang Yuan Garten in Anecdotes of Old Shanghai, S. 98-103 bzw. 159-163. Über die Rolle der Parks für die Entwicklung einer öffentlichen Sphäre siehe v.a. Xiong Yuezhi, S. 336-359. 
die Theater, über deren Aufführungen sie regelmäßig Kritiken schrieben. Daß sich die Reporter auch in den Opiumhallen aufgehalten haben, davon zeugt ein Bericht vor dem Gerichtshof über einen Reporter der Huibao. (North China Herald, 18. Februar 1875)

Die internationalen Berichterstattungen bezogen sich auf Vorgänge und politische Systeme in den fremden Ländern. Schon in den 1870er Jahren behandelten Artikel der Shenbao westliche politische Konzepte wie ,Freiheit', ,Demokratie', ,Präsident' oder ,Parlament' und versuchten deren Inhalte zu erklären. Als beispielhaft für die zahllosen Berichte und Erklärungen über politische Vorgänge und Einrichtungen in westlichen Ländern seien Artikel über die Vorbereitungen zu den amerikanischen Präsidentenwahlen 1878 der Shenbao, Beschreibungen des Besuchs des Ex-Präsidenten Grant in Shanghai oder Diskussionen der Verwendung des Begriffes ,Freiheit' im Westen genannt. ${ }^{25}$ In der Xunhuan Ribao wurde der Eisenbahnbau in Persien diskutiert, oder die englischen und russischen Königsfamilien wurden zu einer ehelichen Verbindung beglückwünscht. ${ }^{26}$ Auch auf der lokalen Ebene waren täglich Nachrichten über aktuelle politische oder ökonomische Vorgänge zu finden, wodurch die Zeitungen eine unschätzbare Quelle zur Erforschung der verschiedensten Aspekte spät-Qingzeitlicher Wirschafts- und Sozialgeschichte sind. Hierunter fallen auch die Gerichtsfalle des Mixed Court oder der Yamen, wie auch Proklamationen der Magistraten oder Daotai.

Ein frühes Beispiel für die Beschäftigung mit lokalpolitischen Angelegenheiten ist die Diskussion um die von westlichen Kaufleuten errichtete Brücke über den Suzhou Creek in Shanghai, die parallel dazu auch in der britischen Tageszeitung North China Daily News geführt wurde. ${ }^{27}$ Streitpunkt in diesem Falle war, daß die Chinesen für das Überqueren der Brücke eine Gebühr entrichten mußten, die westlichen Ausländer aber nicht. ${ }^{28}$ Ein weiterer Teil der

25 „Lun taixiguo shi (Über die Lage in den Westlichen Ländern)“, Shenbao, 12. Januar 1878; Bericht über den Besuch des Ex-Präsidenten Grant in Shanghai, ohne Titel: Shenbao, 23. Mai 1879, S. 4; „Lun xiguo ziyou zhi li, xiang'ai zhi qing (Über die Prinzipien der Freiheit und über die Nächstenliebe in den westlichen Ländern)“, Shenbao, 2. Oktober 1887. Zur Entstehung der politischen Begriffe von „Freiheit", ,Demokratie“ und ,Präsident' im Chinesischen generell siehe Xiong Yuezhi.

26 „Bosi jiang zhu tielu“ (Persien will eine Eisenbahn bauen), Xunhuan Ribao, 4. Februar 1874 (zitiert aus dem ersten Wochenheft); „Shengqi zhihe“ (Zur Gratulation wurde die Flagge gehisst), Xunhuan Ribao, 5. Februar 1874, S. 3.

27 Vgl. die Artikelserie vom 4.-11. Mai 1872 in der North China Daily News. Bei dieser Debatte im Mai 1872 in der Shenbao handelte es sich um die ersten Leserzusendungen der Shenbao überhaupt, wovon der erste sogar möglicherweise von einem westlichen Leser stammen könnte. Die Chinesen werden in diesem Brief mit ,,chinesische Freunde" tituliert, was für einen Chinesen ungewöhnlich wäre.

28 „Ni yi daqiao wei gongqiao yi“ (Vorschlag, aus der Großen Brücke eine Öffentliche Brücke zu machen), Shenbao, 4. Mai 1872, S. 2; „Bian yi gong qiao lun“ (Soll man eine öffentliche Brücke daraus machen?), Shenbao, 9. Mai 1872, S. 2. 
Lokalnachrichten beschäftigte sich mit den neuen westlichen Institutionen in Shanghai und deren Veranstaltungen, aber auch konkret mit der Einführung neuer Technologien in der neuen Großstadt. Diese waren zwar ein fester Teil der Stadtlandschaft Shanghais geworden, erforderten aber immer noch Erklärungen. Der berühmte Zhang Yuan Garten enthielt zum Beispiel ein electric center, in welchem elektrisch betriebene Einrichtungen wie Licht, Klingeln und Ventilatoren eine Attraktion für die Besucher waren. (Anecdotes of Old Shanghai, S. 159-160) Das Gezhi shuyuan führte öffentlich phsyikalische Experimente durch, die in illustrierten Anzeigen angekündigt und - vor allem in illustrierten Berichten der Dianshizhai huabao - besprochen wurden.

Anhand der Selbstdarstellungen der ersten Journalisten der Shenbao läßt sich zumindest skizzenhaft ein Bild der Arbeitsteilung in der Redaktionsstube zeichnen. Der Chefredakteur hatte die zentrale Position inne und war für jede Seite der Zeitung verantwortlich. Er entschied über die Auswahl der Telegramme und Übersetzungen, der Abdrucke aus anderen Zeitungen und selbst der Edikte und Gedichte. Im Falle von Nachrichtenmangel entschied der Chefredakteur über Füll-Artikel oder schrieb selbst Kommentare zu einzelnen Nachrichten oder Einsendungen. Alle Nachrichten wurden ihm vorgelegt, weshalb er letztlich auch für den Abdruck verantwortlich war und zur Rechenschaft gezogen wurde. Wie Ernest Major (im Nekrolog auf seinen Herausgeber Wu Zirang (1818-1878)) feststellte, wurden die meisten der wichtigen Leitartikel vom chinesischen Hauptredakteur geschrieben, die in zwei Fällen zuvor Beamte der Qing-Verwaltung gewesen waren. Offenbar gab es daneben eine Gruppe von Redakteuren, die das größte Interesse am kulturellen Leben Shanghais hatten, sogar selbst dichteten oder Romane schrieben, wie He Guisheng (1840-1894) und Qian Xinbo (1833-?) bzw. später auch Cai Erkang (1852-1920) und Shen Yugui (1807/8-1907). Sie besuchten die Theater, schrieben Kritiken über deren Stücke und waren sehr wahrscheinlich auch die verantwortlichen Redakteure der großen Gedichte-Sektion der Shenbao.

Die schnelle Verbreitung der Tageszeitungen spricht dafür, daß ein großer Bedarf an Informationen aus aller Welt bestand. Der große Erfolg der Shenbao wird häufig ihren zuverlässigen und detaillierten Nachrichten zugeschrieben. ${ }^{29}$ Es wird hervorgehoben, daß die Shenbao als erste Zeitung und schon sehr früh Reporter und Korrespondenten ausgesandt habe, um Nachrichten zu recherchieren. Schon ab 1873 verfolgte sie mit großer Aufmerksamkeit die Vorgänge auf Taiwan und den Ryukyu-Inseln und schickte einen eigenen Korrespondenten nach Taiwan. (Xu Zaiping / Xu Ruifang, S. 343) Zur Berichterstattung des Sino-Französischen Krieges zehn Jahre später schickte sie erstmals einen eigenen Kriegsberichterstatter zum Schauplatz des Geschehens. Für die ,objektive" Berichterstattung, die auch die Niederlagen des chinesischen Mili-

29 Stellvertretend siehe z. B. Link, Kapitel II. 
tärs einschloß, mußte die Shenbao zunächst viel Kritik und den Vorwurf der Parteilichkeit für Frankreich einstecken. Die Auflagenzahlen wurden durch diese Berichte aber enorm gesteigert. ${ }^{30}$ Durch die Inanspruchnahme des Telegraphendienstes war sie seit Anfang der 1880er Jahre den anderen Zeitungen wie der Xinbao und Hubao überlegen. Das alles läßt Roswell Britton feststellen: „The success of the paper was due first to newsservice“ (S. 67).

Dennoch waren Auflagenzahlen aller Tageszeitungen in China im Vergleich zu späteren Entwicklungen insgesamt relativ niedrig. Die britische Daily Press hatte 1874 eine Auflage von 400-500 Exemplaren, die Hongkong Times sogar nur $190 .{ }^{31}$ Diese britischen Zeitungen wandten sich allerdings auch an eine wesentlich kleinere Lesergemeinschaft von Ausländern in der Kolonie. Die Huazi Ribao gab zu ihrer Gründung 1872 die ehrgeizige Aussicht auf eine Auflage von 3000 bis 4000 Ausgaben an. (China Mail, 2. April 1872) Zwei Jahre später garantierte sie ,an eventual circulation of one thousand copies“ (China Mail, 24. Februar 1874).

Für die erste rein chinesischsprachige Tageszeitung Xunhuan Ribao varrieren die Zahlen zwischen 500 bis 700 Ausgaben (Lin Youlan, S. 13), während die statistischen Jahrbücher des Colonial Office in Hongkong von 1000 bis 1200 Exemplare im Jahre 1902 registrieren.

Nur die Shenbao versammelte schon nach wenigen Monaten eine enorme Leserschaft um sich. Schon im Juni 1872 konnte sie stolz verkünden, daß sie die Auflagenzahl auf 4500 pro Tag erhöhen konnte, so daß es nun ,in Shanghai keinen Gelehrten oder Kaufmann mehr gibt, der die Zeitung nicht liest." ${ }^{\text {"32 }}$ Bei einer geschätzten Zahl von 200000 chinesischen Einwohnern in den Shanghaier Konzessionsgebieten ist diese Aussage als leichte Übertreibung einzuschätzen. (Murphey, S. 22) Doch ist dabei ins Gedächtnis zu rufen, daß die Auflagenzahlen nicht mit den Leserzahlen identisch sind. So ist in Sekundärquellen zu lesen, daß in China um die zehn Leser auf ein Zeitungsexemplar gerechnet werden könnten. ${ }^{33}$ Wenn man den Anzeigen von 1877 Glauben schenken mag, in der von Auflagen von 8000-9000 (Shenbao, 20. Juni 1877) und sogar bis zu 10000 Exemplaren (Shenbao, 10. Februar 1877) gesprochen wird, und man dies mit einem niedrig angesetzten Verteilungsfaktor von durchschnittlich fünf Lesern multipliziert, wäre tatsächlich fast ein Viertel der chinesischen Bevölkerung Shanghais Leser dieser Zeitung gewesen. Diese Auflagenzahlen sind im Vergleich zu allen anderen chinesischen Zeitungen der

30 Britton, S. 67-68; Xu Zaiping, S. 125-133.

31 Great Britain Colonial Office. Blue Books, Hongkong, 1874: „Return of the Principal Publications in 1874“. Für die Daily Press werden keine Angaben gemacht.

32 Ohne Titel, Shenbao, 11. Juni 1872. S. 1 und auch an folgenden Tagen. Zum Vergleich: Die Auflagenzahl der Xunhuan Ribao liegt erst im Jahr 1901 bei 1200 Ex. Die Angaben der Sekundärliteratur über die ersten Jahre sprechen von 400 bis 600 Exemplaren.

33 Britton spricht von 10-20 Lesern pro Exemplar eines Magazines. Roswell Britton, [1933]. S. 129. Patterson nennt 12-15. Patterson, S. 58. 
Zeit sehr hoch - decken sich aber in etwa mit den Ausführungen des britischen Konsuls Walter H. Medhurst Jr. (1823-1885) von 1876, der sie direkt von Major selbst erfahren hatte.

Zusammenfassend läßt sich sagen, daß die moderne chinesische Presse im 19. Jahrhundert nicht nur einen öffentlichen Diskurs über aktuelle und zentrale wirtschaftliche, politische und gesellschaftliche Themen eröffnet, sondern auch auf unterhaltsame Weise neue moralische und gesellschaftliche Werte im sozialen Umgang eingeführt und vertraut gemacht hat. In diesen Diskurs involviert war ein breites Spektrum von aufgeschlossenen chinesischen und ausländischen Beamten, Unternehmern, Missionaren und Literaten, die sich auf innovativen Wegen über die krisenerschütterte Gesellschaft austauschten. Die Einführung von neuem Wissen über die Welt spielte in diesen Debatten auch in China eine besondere Rolle. Die vielfältigen Kollaborationen zwischen europäischen und chinesischen Journalisten, Wissenschaftlern und Übersetzern machten diese Gruppe von Akteuren für Europa zum zentralen Vermittler von Wissen über China im 19. Jahrhundert.

\section{Anhang \\ Zitierte Zeitungen}

\section{Shenbao}

4. Mai (1872) 2 - Ni yi daqiao wei gongqiao yi (Vorschlag, aus der Großen Brücke eine Öffentliche Brücke zu machen).

9. Mai (1872) 2 - Bian yi gong qiao lun (Soll man eine öffentliche Brücke daraus machen?).

7. Juli (1875) 1 - Yan you fangshi gaobai (Reporter gesucht).

20 Februar (1877) - Yan qing tongren (Übersetzer gesucht).

20. Juni (1877) 1 - Benguan zishu jiazeng (Erweiterung der Zeichenanzahl unserer Zeitung).

12. Januar (1878) - Lun taixiguo shi (Über die Lage in den Westlichen Ländern).

23. Mai (1879) - [ohne Titel]: Bericht über den Besuch des Ex-Präsidenten Grant in Shanghai.

11. August (1886) 1 - Lun xinwenzhi zhi yi (Über den Vorteil von Zeitungen).

2. Oktober (1887) - Lun xiguo ziyou zhi li, xiang'ai zhi qing (Über die Prinzipien der Freiheit und über die Nächstenliebe in den westlichen Ländern).

\section{Xunhuan Ribao}

4. Februar (1874) 4 - Bosi jiang zhu tielu (Persien will eine Eisenbahn bauen), (zitiert aus dem ersten Wochenheft).

5. Februar (1874) 3 - Benqu ribao tongqi (Anzeige unsere Nachrichtenhauses).

5. Februar (1874) 3 - Shengqi zhihe (Zur Gratulation wurde die Flagge gehisst).

6. Februar (1874) 3 - Ribao you bi yu shi zhengzhi (Zeitungen sind für die Tagespolitik von Nutzen). 


\section{China Mail}

2. April (1872) - New Publication.

2. Feb (1874) - Prospectus.

24. Februar (1874) - Notice.

\section{North China Herald}

18. Februar (1875) - Law report.

\section{Baoxue Zazhi}

Vol. 1. no. 10 (Januar 1949) - Jizhe zhe xie (Die Schuhe der Journalisten).

\section{Literaturverzeichnis}

Anecdotes of Old Shanghai, Shanghai (Shanghai Cultural Publishing House) 1985.

Barnett, Suzanne W.: Silent Evangelism: Presbyterians and the Mission Press in China. 1807-1860, in: Journal of Presbyterian History, 49. 9 (1971), 287-302.

Bartlett, Beatrice: Monarchs and Ministers: the Grand Council in Mid-Ch'ing China, 17231820, Berkely, Los Angeles \& Oxford (University of California Press ) 1991.

Bennett, Adrian A.: Missionary Journalist in China. Young Allen and His Magazines, 1860-1883, Athens (University of Georgia Press) 1983.

-: John Fryer: The Introduction of Western Science and Technology into Nineteenth Century China, Cambridge, MA (Harvard Univiversity Press) 1967.

Biggerstaff, Knight: Shanghai Polytechnic Institution and Reading Room: An Attempt to Introduce Western Science and Technology to the Chinese, in: The Pacific Historical Review, vol. XXV (1956), 127-149.

Britton, Roswell: The Chinese Periodical Press, 1800-1912, Shanghai (Kelly \& Walsh) [1933], (Reprint: Taipei: Ch'eng-wen Publishing Co., 1966).

Cohen, Paul: Between Tradition and Modernity. Wang T'ao and Reform in Late Ch'ing China. Cambridge, MA \& London (Harvard University Press) 1987.

Eastman, Lloyd: Ch'ing-i and Chinese Policy Formation During the Nineteenth Century, in: Journal of Asian Studies, 24. 4. (1965), 595-611.

Fang Hanqi (Hg.): Zhongguo xinwen shiye tongshi, juan yi. (Allgemeine Geschichte des chinesischen Journalismus, vol 1) Beijing (Zhongguo renmin daxue chubanshe) 1992.

Guo Weidong (Hg.): Jindai waiguo zai Hua wenhua jigou zonglu (Gesamtliste der ausländischen Kulturinstitutionen im modernen China), Shanghai (Shanghai renmin chubanshe) 1993.

Jacobi, R.: Der Journalist, Hannover 1902.

Ji Shaofu (Hg): Zhongguo chuban jianshi (Kurze Geschichte des Publikationswesens in China), Shanghai (Xuelin chubanshe) 1991.

King, Frank H. H. \& Clarke, Prescott: A Research Guide to China-Coast Newspapers, 1822-1911, Cambridge, MA (Harvard University Press) 1965 (Harvard East Asian Monographs). 
Liang Qichao: Lun baoguan you yi yu guoshi (Über den Nutzen der Zeitungen für die Staatsangelegenheiten) in: Shiwubao, 1 (9. August 1896). Reprint: Zhongguo jindai baokan shi cankao ziliao, shang (Referenzmaterialien zur modernen Zeitungsgeschichte Chinas, vol. 1) Beijing, 1982, 242-245.

Lei Jin: Shenbaoguan zhi guoqu zhuangkuang (Die Lage des Shenbao-Verlages in der Vergangenheit), in: Zuijin zhi wushi nian. Shenbao guan wu shi zhou jinian (Die letzten 50 Jahre. Jubiläumsband zum 50jährigen Bestehen der Shenbao), Shanghai (Shanghai shudian), 1987 (Reprint), Teil III, 26-28.

Li San-pao: Letters to the Editor in Fryer's Scientific Magazine 1877-1892. An Analysis, in: Zhongyang yanjiuyuan jindaishi yanjiu, IV.2 (1974), 729-777.

Lin Youlan: Xianggang baoye fazhan shi (Geschichte der Entwicklung des Hongkonger Zeitungswesens), Xianggang (Shijie shuju) 1977.

Link, Perry: Mandarin Ducks and Butterflies. Popular Fiction in Early Twentieth-Century Chinese Cities, Los Angeles \& London (University of California Press) 1981.

Lo Hsiang-lin: The Role of Hongkong in the Cultural Interchange Between East and West Vol I - II, Tokyo, 1963 (Center for East Asian Cultural Studies, Ser. No 6).

Lu Yongling \& Hayhoe, Ruth: Chinese Higher Learning: The Transition Process from Classical Knowledge Patterns to Modern Disciplines, 1860-1910, in: Christoph Charle \& Jürgen Schriewer (Hgg.), Transnational Intellectual Networks and the Cultural Logic of Nations, Providence, RI \& Oxford (Bergham) 1998.

Memorials of Protestant Missionaries to the Chinese: Giving a List of their Publications, and Obituary Notices of the Deceased, Shanghai (American Presbyterian Mission Press), 1867 (Repr: Taipei: Chengwen, 1967).

Murphey, Rhoads: Shanghai-Key to Modern China, Cambridge, MA (Harvard University Press) 1953.

O'Boyle, Lenore: The Image of the Journalist in France, Germany, and England, 1815-1848, in: Comparative Studies in Society and History, 10 (1968), 291-317.

Oebsger-Roeder, Rudolf: Untersuchungen über den Bildungsstand der deutschen Journalisten, Leipzig: (Universitätsverlag) 1936 (Wesen und Wirkungen der Publizistik vol. 7).

Patterson, Don: The Journalism of China, in: The University of Missouri Bulletin, 23.34 (1922) (Monograph Series).

Sinn, Elizabeth: Fugitive in Paradise: Wang Tao and His Life in Hongkong. A Study in Cultural Transformation, (Unveröffentlichtes Manuskript vorgelegt zur ,James Legge“"Konferenz, University of Aberdeen, 8-12 April) 1997.

Smith, Carl T.: The English-educated Chinese Elite in Nineteenth-Century Hong Kong, in: David Faure (Hgg.), History of Hong Kong 1842 -1984, Hongkong \& London (Tamarind Books) 1995, 29-56.

Soothill, William E: Timothy Richard of China. Seer, Statesman, Missionary and the Most Disinterested Adviser the Chinese Ever Had, London (Seeley, Service \& Co.) 1924.

Tsou Mingteh: Christian Missionary as Confucian Intellectual: Gilbert Reid (18571927) and the Reform Movement in the Late Qing, in: Daniel Bays (Hg.), Christianity in China. From the Eighteenth Century to the Present, Stanford, CA (Stanford University Press) 1996, 73-90. 
Vittinghoff, Natascha: Fruchtbares Neuland: Das kulturelle und politische Experimentierfeld Hongkong im 19. Jahrhundert, Das Neue China, 24.2 (1997), 23-25.

Wagner, R.G.: Joining the Global Imaginaire: The Shanghai Illustrated Newspaper Dianshizhai huabao, in: Joining the Global Public. Word, Image and City in the Early Chinese Newspapers 1870-1910, New York (SUNY) 2007, 105-174.

Woodbridge, S. I.: A Pioneer and Exponent of Journalism in China. The ,Sin Wan Pao', in: Special Supplement of the Sin Wan Pao. On the Occasion of the $30^{\text {th }}$ Anniversary of the Foundation of the Paper. 1893-1923, Shanghai (Sin Wan Pao Company) 1922, 31-33.

Xiong Yuezhi: Xixue Dongjian yu wan Qing shehui. (Der Fluß westlicher Wissenschaften nach Osten und die Gesellschaft der späten Qing), Shanghai (Shanghai Renmin chubanshe) $1995^{2}$.

-: Zhang Yuan: Wan Qing Shanghai yige gonggong kongjian yanjiu (Der Zhang Garten: Forschungen zu einem öffentlichen Raum im Shanghai der späten QingZeit), in: Zhang Zhongli (Hrsg), Zhongguo jindai chengshi qiye, shehui, kongjian (Unternehmen, Gesellschaft und öffentliche Räume in der modernen chinesischen Stadt), Shanghai (Shanghai shehui kexue chubanshe) 1999, 336-359.

-: The Translation and Usage of Some Political Terms in Late Qing China, in: Natascha Vittinghoff (Übers), in: Iwo Amelung, Joachim Kurtz und Michael Lackner (Hgg.), New Terms for New Ideas: Studies in the Formation of Modern Chinese Scientific and Cultural Terminologies (I), Brill (Leiden) 2001, 69-93.

Xu Zaiping \& Xu Ruifang: Qingmo sishinian Shenbao shiliao (Historisches Material zu 40 Jahren Shenbao in der späten Qing-Zeit), Beijing (Xinhua chubanshe) 1988.

Zhou Jiarong: Zai Xianggang yu Wang Tao huimian - Zhong Ri liangguo mingshi de fang Gang jilu (Wiedersehen mit Wang Tao in Hongkong - Aufzeichnungen von Hongkongbesuchen zelebrierter Gelehrter aus China und Japan), in: Lam Kai Yin \& Wong Man Kong (Hgg.) Wang Tao yu jindai shijie (Wang Tao und die moderne Welt), Hongkong 1997, 375-394. 